International Mathematical Forum, 2, 2007, no. 22, 1053 - 1068

\title{
On Certain Classes of Degree Reverse Lexicographic Gröbner Bases
}

\author{
Gaetana Restuccia \\ Dipartimento di Matematica, Università di Messina \\ Contrada Papardo, salita Sperone, 31 \\ 98166 Messina, Italy \\ e-mail:grest@dipmat.unime.it \\ Giancarlo Rinaldo \\ Dipartimento di Matematica, Università di Messina \\ Contrada Papardo, salita Sperone, 31 \\ 98166 Messina, Italy \\ e-mail:rinaldo@dipmat.unime.it
}

\begin{abstract}
We prove for a standard graded $K$-algebra $A=R / I$, where $K$ is a field, $R$ the polynomial ring $K\left[x_{1}, \ldots, x_{n}\right]$, that, if $I$ has a universal degree reverse lexicographic Gröbner basis of degree 2, then $A$ is a strongly Koszul algebra. If $A$ is a homogeneous semigroup ring, the same result is obtained by using the Graver bases theory.
\end{abstract}

Mathematics Subject Classification: 13D02, 13P10

Keywords: Gröbner basis, Graver basis, semigroup ring, linear resolution, Koszul algebra 


\section{Introduction}

Let $R=K\left[x_{1}, \ldots, x_{n}\right]$ be the polynomial ring over the field $K$ and $A=R / I$ be the standard graded $K$-algebra where $I$ is a graded ideal. It is known that the $K$-algebra $A$ is Koszul if the residue field $K$ has a linear resolution as an $A$-module.

It is easy to see that if $A$ is a Koszul algebra then $I$ is generated by quadratic polynomials. From the other hand, if $I$ has a quadratic Gröbner basis with respect to some term order, then $A$ is Koszul (see [3]).

In general these implications are strict.

The definition of strongly Koszul algebra is given in [6]. More precisely a homogeneous $K$-algebra, is called strongly Koszul if it is a finitely generated graded $K$-algebra which is generated over $K$ by elements of degree 1 and its graded maximal ideal $\mathfrak{m}$ admits a minimal system of generators $u_{1}, \ldots, u_{n}$ such that for all subsequences $u_{i_{1}}, \ldots, u_{i_{r}}$ with $i_{1}<i_{2}<\cdots<i_{r}$ and for all $j=1, \ldots, r-1$, the colon ideal $\left(u_{i_{1}}, \ldots, u_{i_{j-1}}\right): u_{i_{j}}$ is generated by a subset of elements of $\left\{u_{1}, \ldots, u_{n}\right\}$.

The condition " $I$ has a Gröbner basis of degree 2" is not sufficient for $A$ to be a strongly Koszul algebra.

Consider the universal degree reverse lexicographic Gröbner basis $\left(U_{d r}\right.$ for short), as the union of the Gröbner basis $G_{i}$

$$
\bigcup_{i=1}^{n !} G_{i}
$$

where every $G_{i}$ is defined with respect to one of the $n$ ! degree reverse lexicographic order induced by all permutations of variables $x_{1}, \ldots, x_{n}$.

In section 1 we prove that for any graded standard $K$-algebra, if the universal degree reverse lexicographic Gröbner basis of $I$ is of degree 2, then $A$ is strongly Koszul. We use essentially a result obtained in [3], where the authors consider the Koszul property of a standard graded algebra $A$, deduced by the existence of a Gröbner basis of degree 2, for the ideal $I$ of $R$, $A=R / I=K\left[\tilde{x}_{1}, \ldots, \tilde{x}_{n}\right], \tilde{x}_{i}=x_{i}+I$.

The crucial point is that any ideal $\left(\tilde{x}_{l}, \ldots, \tilde{x}_{n}\right)$ with $1 \leq l \leq n$ has a linear resolution if the term order in $R$ is the reverse lexicographic one. 
Since we have $n$ ! reverse lexicographic orderings on the monomials of $R$, depending on the permutation of the variables, this fact induces a strong condition about Gröbner basis of $I$ that gives the strongly Koszul property of $A$.

In section 2 the notion of strongly Koszul algebras is studied when $A=R / I$ is a homogeneous semigroup algebra. We recall the definition of Graver basis and primitive binomials of $I$ and we establish a bound for the degree of the generators of the ideals $\left(\tilde{x}_{i}\right) \cap\left(\tilde{x}_{j}\right)$, for all $i \neq j$, that is given by the maximum degree of the primitive binomials in $I$. If this maximum degree is 2 , then $A$ is strongly Koszul. At the end of this section we give a alternative proof of the result given in section 1 in the case of semigroup algebra, using properties of the primitive binomials that are elements of the universal degree reverse lexicographic Gröbner basis of degree 2.

The last section is dedicated to the research of the universal degree reverse lexicographic Gröbner basis of two classical semigroup algebras that are strongly Koszul (see [6]):

1. The 2th Veronese algebra;

2. The Segre product of two polynomial rings.

For these purposes we consider the indexes appearing in the monomial of each primitive binomial in the ideal of presentation $I$ of $A$ and we fix an "ordering" related to these indexes. As expected, the universal degree reverse lexicographic Gröbner bases of these algebras have degree 2.

\section{Universal degree reverse lexicographic Gröbner basis}

Let $R=K\left[x_{1}, \ldots, x_{n}\right]$ be the polynomial ring in $n$ variables on a field $K$, $I \subset R$ a graded ideal.

We recall the

Definition 1.1. A finite subset $U \subset I$ is called a universal Gröbner basis if $U$ is a Gröbner basis of I with respect to all term orders simultaneously. 
Now we consider a particular subset of the universal Gröbner basis

Definition 1.2. Let $I$ be an homogeneous ideal of the polynomial ring $R=$ $K\left[x_{1}, \ldots, x_{n}\right]$. We call universal degree reverse ( $U_{d r}$ for short), lexicographic Gröbner basis the union of the Gröbner basis $G_{i}$

$$
\bigcup_{i=1}^{n !} G_{i}
$$

where every $G_{i}$ is defined with respect to one of the $n$ ! degree reverse lexicographic order induced by

$$
x_{i_{1}}>\cdots>x_{i_{n}}
$$

with $\left\{i_{1}, \ldots, i_{n}\right\}=\{1, \ldots, n\}$.

Example 1.3. Let $R$ the polynomial ring in $2 m$ variables

$$
K\left[x_{11}, \ldots, x_{1 m}, x_{21}, \ldots, x_{2 m}\right]
$$

and let I be the ideal generated by the set of binomials

$$
F=\left\{x_{1 i} x_{2 j}-x_{1 j} x_{2 i} \mid 1 \leq i<j \leq m\right\}
$$

Then $F$ is a universal Gröbner basis for $I$.

Proof. See [7], Example 1.4.

Example 1.4. If $I$ is a monomial ideal then the unique minimal set of generators of $I$ is a universal Gröbner basis for $I$.

Example 1.5. Let $R=K\left[x_{1}, \ldots, x_{n}\right]$ be a polynomial ring and $I$ a principal ideal generated by a form $f$. Then $\{f\}$ is the universal Gröbner basis for the ideal $I$.

In the previous examples the universal Gröbner basis and the universal degree lexicographic Gröbner basis coincide, and in particular they have the same degree.

This is not always the case as the following example shows. 
Example 1.6. Let

$$
\mathcal{S}=\left\{x_{1}^{2}, x_{1} x_{2}, x_{1} x_{3}, x_{2}^{2}, x_{2} x_{3}, x_{3}^{2}\right\}
$$

be a set of monomials in $R=K\left[x_{1}, x_{2}, x_{3}\right]$, and let $K[\mathcal{S}]$ the monomial subring spanned by $\mathcal{S}$ :

$$
K[\mathcal{S}]=K\left[x_{1}^{2}, x_{1} x_{2}, x_{1} x_{3}, x_{2}^{2}, x_{2} x_{3}, x_{3}^{2}\right] \subset R
$$

The presentation ideal I of $K[\mathcal{S}]$ in the ring $K\left[t_{1}, \ldots, t_{6}\right]$ defines the Veronese surface in the projective space $\mathbb{P}^{5}$.

The universal Gröbner basis is given by the set of binomials of degree 2,

$$
F_{2}=\left\{t_{2} t_{3}-t_{1} t_{5}, t_{3} t_{4}-t_{2} t_{5}, t_{3} t_{5}-t_{2} t_{6}, t_{3}^{2}-t_{1} t_{6}, t_{2}^{2}-t_{1} t_{4}, t_{5}^{2}-t_{4} t_{6}\right\}
$$

and by the binomials of degree 3 ,

$$
F_{3}=\left\{t_{3}^{2} t_{4}-t_{1} t_{5}^{2}, t_{1} t_{5}^{2}-t_{2}^{2} t_{6}, t_{3}^{2} t_{4}-t_{2}^{2} t_{6}\right\}
$$

The universal degree reverse lexicographic Gröbner basis is given only by the binomials in $F_{2}$.

Theorem 1.7. Let I be an ideal of $R=K\left[x_{1}, \ldots, x_{n}\right]$ which has a Gröbner basis of homogeneous elements of degree 2 with respect to a given term order. Then

1. $A=R / I$ is a Koszul algebra;

2. more generally, if $\operatorname{in}\left(I+\left(x_{i_{1}}, \ldots, x_{i_{l}}\right)\right)=\operatorname{in}(I)+\left(x_{i_{1}}, \ldots, x_{i_{l}}\right)$, then $A / J$ has a linear resolution for the ideal $J=\left(\tilde{x}_{i_{1}}, \ldots, \tilde{x}_{i_{l}}\right), 1 \leq i_{1} \leq \cdots \leq$ $i_{l} \leq n$, with $\tilde{x}_{i}=x_{i}+I, \forall i, 1 \leq i \leq n$.

Proof. See [3], Theorem 2.2.

Proposition 1.8. If I has a Gröbner basis of degree 2 with respect to the reverse lexicographic term ordering, then $A /\left(\tilde{x}_{l}, \ldots, \tilde{x}_{n}\right)$ has a linear A-resolution with $1 \leq l \leq n$.

Proof. See [3], Corollary 2.5. 
Definition 1.9 ([6]). The homogeneous $K$-algebra $A$ is called strongly Koszul if its graded maximal ideal $\mathfrak{m}$ admits a minimal system of homogeneous generators $u_{1}, \ldots, u_{n}$ such that for all subsequences $u_{i_{1}}, \ldots, u_{i_{r}}$ with $i_{1}<i_{2}<\cdots<$ $i_{r}$ and for all $j=1, \ldots, r-1$, the colon ideal $\left(u_{i_{1}}, \ldots, u_{i_{j-1}}\right): u_{i_{j}}$ is generated by a subset of elements of $\left\{u_{1}, \ldots, u_{n}\right\}$.

Theorem 1.10. Let $R=K\left[x_{1}, \ldots, x_{n}\right]$ be the polynomial ring and $I$ a graded ideal of $R$. Suppose that $I$ admits a universal degree reverse lexicographic Gröbner basis of degree 2.

Then $A / I$ is a strongly Koszul algebra.

Proof. By Proposition 1.8 the $A$-module $A /\left(\tilde{x}_{l}, \ldots, \tilde{x}_{n}\right)$ has a linear $A$-resolution since $I$ has a reverse lexicographic Gröbner basis of degree 2.

Since $I$ has a universal degree reverse lexicographic Gröbner basis of degree 2 we easily obtain that $A /\left(\tilde{x}_{i_{1}}, \ldots, \tilde{x}_{i_{s}}\right)$ has a linear $A$-resolution for all subset $\left\{\tilde{x}_{i_{1}}, \ldots, \tilde{x}_{i_{s}}\right\}$ of the set $\left\{\tilde{x}_{1}, \ldots, \tilde{x}_{n}\right\}$ by changing the order of $x_{i}$ and applying Proposition $1.8 n$ ! times.

Now since the matrices of the $A$-resolution have linear entries it is easy to observe that also the colon ideals

$$
\left(\tilde{x}_{i_{1}}, \ldots, \tilde{x}_{i_{s}}\right): \tilde{x}_{i_{s+1}}
$$

for all $1 \leq i_{1} \leq \cdots \leq i_{s+1} \leq n$, are generated by subsets of $\tilde{x}_{1}, \ldots, \tilde{x}_{n}$. The assertion follows by the definition of strongly Koszul algebra.

Remark 1.11. Assuming that in the examples 1.3, 1.4, 1.5, the generators of the ideals have degree 2 one can see that these algebras are strongly Koszul and their universal Gröbner basis is of degree 2. Therefore a natural question is if strongly Koszul implies universal Gröbner basis of degree 2.

Example 1.12. If we consider $K\left[x_{1}^{2}, x_{1} x_{2}, x_{2}^{2}, x_{2} x_{3}, x_{3}^{2}\right]=R / I$, we see that $I$ is generated by quadratic Gröbner basis, therefore is Koszul, but is not strongly Koszul. Its universal degree reverse lexicographic Gröbner basis has an element of degree 3 :

$$
U_{d r}(\mathcal{S})=\left\{t_{1} t_{3}-t_{2}^{2}, t_{3} t_{5}-t_{4}^{2}, t_{5} t_{2}^{2}-t_{1} t_{4}^{2}\right\}
$$




\section{$2 \quad U_{d r}$ of degree 2 implies strongly Koszul}

In this section we prove Theorem 1.10 in the case $A$ is a semigroup ring, by using the theory of Graver basis of $I$.

Let $R=K\left[x_{1}, \ldots, x_{n}\right]$ be a polynomial ring over a field $K$ and let $\mathcal{S}=$ $\left\{f_{1}, \ldots, f_{r}\right\}$, with $f_{i}$ monomials in $R$. For each monomial $f \in R, \log (f)$ is the multi-index of $f$.

The subring of $A$ generated by $\mathcal{S}$

$$
K[\mathcal{S}]=K\left[f_{1}, \ldots, f_{r}\right]=K\left[\left\{x^{\alpha} \mid \alpha \in \mathbb{N} \log \left(f_{1}\right)+\cdots+\mathbb{N} \log \left(f_{r}\right)\right\}\right]
$$

is called semigroup ring, induced by the semigroup $\mathbb{N} \log \left(f_{1}\right)+\cdots+\mathbb{N} \log \left(f_{r}\right)$.

Let $\varphi$ be the graded epimorphism of $K$-algebras

$$
\varphi: B=K\left[T_{1}, \ldots, T_{r}\right] \longrightarrow K[\mathcal{S}]
$$

with $\varphi\left(T_{i}\right)=f_{i}$ and $\operatorname{deg}\left(T_{i}\right)=\operatorname{deg}\left(f_{i}\right), i=1, \ldots, r$. The kernel of $\varphi, P(\mathcal{S})$, that is a graded prime ideal generated by binomials (see [8], Proposition 7.1.2), is called toric ideal of $K[\mathcal{S}]$.

Definition 2.1. Let $F=\left\{f_{1}, \ldots, f_{r}\right\}$ be a finite set of monomials in a polynomial ring $K\left[x_{1}, \ldots, x_{n}\right]$, and let $P(\mathcal{S})$ the toric ideal of $K[\mathcal{S}]$.

A binomial

$$
T^{\alpha}-T^{\beta} \in P(\mathcal{S})
$$

with $\alpha, \beta \in \mathbb{N}^{r}$, is called primitive if there exists no other binomial $T^{\delta}-T^{\gamma} \in P$ such that $T^{\delta}$ divides $T^{\alpha}$ and $T^{\gamma}$ divides $T^{\beta}$.

The set of all primitive binomials in $P(\mathcal{S})$ is called Graver basis $\operatorname{Gr}_{\mathcal{S}}$ of $P(\mathcal{S})$.

Remark 2.2. The Universal Gröbner basis of $P(\mathcal{S})$ (that is the Gröbner basis with respect to all term orders) is a subset of the Graver basis $\operatorname{Gr}_{\mathcal{S}}$ (see [7], Chapter 7).

Definition 2.3. Let $R=K\left[x_{1}, \ldots, x_{n}\right]$ the polynomial ring and $<$ a term order on $R$. If $f=x^{\alpha}-y^{\beta}$ is a binomial in $A$ then we call initial term the bigger term with respect to the term order $<$ and we call trailing term the other one. 
Let assume $K[\mathcal{S}]$ be an homogeneous semigroup ring generated by the monomials $F=\left\{f_{1}, \ldots, f_{r}\right\} \subset R$ of the same degree.

We fix the degree of every generator of the graded maximal irrelevant ideal $\mathfrak{m}$ of $K[\mathcal{S}]$ to 1 .

Proposition $2.4([6])$. Let $K[\mathcal{S}]=K\left[u_{1}, \ldots, u_{r}\right]$ be an homogeneous semigroup with graded maximal ideal $\mathfrak{m}=\left(u_{1}, \ldots, u_{r}\right)$. Then $K[\mathcal{S}]$ is a strongly Koszul algebra if and only if the ideals $\left(u_{i}\right) \cap\left(u_{j}\right)$ are generated in degree 2 , for all $i \neq j$.

Let $\delta(I)$ be the maximum degree of the minimal set of generators of a monomial ideal $I$ of the $\operatorname{ring} A$.

Proposition 2.5. Let $K[\mathcal{S}]$ be a semigroup ring and let $u_{1}, \ldots, u_{r}$ be the generators of $K[\mathcal{S}]$. Then, for all $i \neq j$, one has $2 \leq \delta\left(\left(u_{i}\right) \cap\left(u_{j}\right)\right) \leq k$, where $k$ is the maximum degree between the primitive binomial in $\mathrm{Gr}_{\mathcal{S}}$ involving $u_{i}$ and $u_{j}$.

If there is not a primitive binomial involving both $u_{i}$ and $u_{j}$, then $\delta\left(u_{i}, u_{j}\right)=$ 2.

Proof. Let $a$ be in the minimal set of generators of $\left(u_{i}\right) \cap\left(u_{j}\right)$ and we write

$$
a \cong u_{i} u^{\alpha} \cong u_{j} u^{\beta}
$$

with $\alpha$ and $\beta$ in $\mathbb{N}^{r}$.

Suppose that $T_{i} T^{\alpha}-T_{j} T^{\beta} \in P(\mathcal{S})$ is not a primitive binomial. This implies that exists a primitive binomial $T^{\gamma}-T^{\delta} \in \mathrm{Gr}_{\mathcal{S}}$ such that $T^{\gamma} \mid T_{i} T^{\alpha}$ and $T^{\delta} \mid T_{j} T^{\beta}$.

Then we have one of the following cases:

i) $T_{i} \backslash T^{\gamma}$ and $T_{j} \not T^{\delta}$;

ii) $T_{i} \mid T^{\gamma}$ and $T_{j} \mid T^{\delta}$;

iii) $T_{i} \mid T^{\gamma}$ and $T_{j} \not T^{\delta}$.

i) $T_{i} \backslash T^{\gamma}$ and $T_{j} \not T^{\delta}$ implies that $T^{\gamma} \mid T^{\alpha}$ and $T^{\delta} \mid T^{\beta}$ and we have $T_{i} T^{\alpha}-T_{j} T^{\beta}-T^{\alpha-\gamma}\left(T_{i} T^{\gamma}-T_{i} T^{\delta}\right)=T_{i} T^{\delta} T^{\alpha-\gamma}-T_{j} T^{\beta}$

$$
=T^{\delta}\left(T_{i} T^{\alpha-\gamma}-T_{j} T^{\beta-\delta}\right) .
$$


Since $P(\mathcal{S})$ is a prime ideal $T_{i} T^{\alpha-\gamma}-T_{j} T^{\beta-\delta} \in P(\mathcal{S})$ and $a$ is not a generator of $\left(u_{i}\right) \cap\left(u_{j}\right)$.

ii) If $T_{i} \mid T^{\gamma}$ and $T_{j} \mid T^{\delta}$ we have $u^{\gamma} \cong u^{\delta} \in\left(u_{i}\right) \cap\left(u_{j}\right)$ and since $u^{\gamma} \cong u^{\delta} \mid a, a$ is not a generator of $\left(u_{i}\right) \cap\left(u_{j}\right)$.

iii) If $T_{i} \mid T^{\gamma}$ and $T_{j} \backslash T^{\delta}$ we have $T^{\delta} \mid T^{\beta}$, therefore

$$
u_{i} u^{\alpha} \cong u_{j} u^{\beta} \cong u_{j} \frac{u^{\beta}}{u^{\delta}} u^{\gamma}
$$

and $u_{i} u_{j} \mid u_{j} \frac{u^{\beta}}{u^{\delta}} u^{\gamma}$, therefore $a$ is not a generator.

From Proposition 2.5 follows immediately

Corollary 2.6. Let $K[\mathcal{S}]$ be a homogeneous semigroup ring and let $u_{1}, \ldots, u_{r}$ be the generators of $K[\mathcal{S}]$. If for all $i \neq j$, one has $\delta\left(\left(u_{i}\right) \cap\left(u_{j}\right)\right)=k=2$, where $k$ is the maximum degree between the primitive binomial in $\operatorname{Gr}_{\mathcal{S}}$ involving $u_{i}$ and $u_{j}$, then $K[\mathcal{S}]$ is a strongly Koszul algebra.

Theorem 2.7. If $P(\mathcal{S})$ has a universal degree reverse lexicographic Gröbner basis of degree 2 , then $K[\mathcal{S}]$ is strongly Koszul.

Proof. Let $K[\mathcal{S}]=K\left[u_{1}, \ldots, u_{r}\right]$. We want to show that $\forall i \neq j=1, \ldots, r$, $\left(u_{i}\right) \cap\left(u_{j}\right)$ is generated by monomials of degree 2 .

By Proposition 2.5 it follows

$$
\left(u_{i}\right) \cap\left(u_{j}\right)=\left(u_{i} u_{j}, u_{i} u^{\alpha_{1}} \cong u_{j} u^{\beta_{1}}, \ldots, u_{i} u^{\alpha_{s}} \cong u_{j} u^{\beta_{s}}\right) .
$$

and $T_{i} T^{\alpha_{1}}-T_{j} T^{\beta_{1}}, \ldots, T_{i} T^{\alpha_{s}}-T_{j} T^{\beta_{s}}$ are primitive binomials.

This implies that the minimal set of generators of $\left(u_{i}\right) \cap\left(u_{j}\right)$ is a subset of

$$
I=\left\{u_{i} u_{j}, u_{i} u^{\alpha_{1}} \cong u_{j} u^{\beta_{1}}, \ldots, u_{i} u^{\alpha_{s}} \cong u_{j} u^{\beta_{s}}\right\}
$$

Therefore it is sufficient to show that all elements in $I$ of degree bigger than two are generated by elements of degree 2 in $I$.

Case 1) Suppose that there are not primitive binomials of degree 2 such that $T_{i} T_{k}-T_{j} T_{k^{\prime}} \in P(\mathcal{S})$. We want to show that $\left(u_{i}\right) \cap\left(u_{j}\right)=\left(u_{i} u_{j}\right)$.

Let $u_{i} u^{\alpha} \cong u_{j} u^{\beta}$ of degree $>2$. This implies $T_{i} T^{\alpha}-T_{j} T^{\beta} \in P(\mathcal{S})$. We fix a degree reverse lexicographic ordering such that $T_{j}<T_{i}<T_{k} \forall k=1, \ldots, r, k \neq$ $i, k \neq j$. 
By hypothesis, the Gröbner basis of $P(\mathcal{S})$ has degree 2 and if $T_{j}$ divides one of the monomials in a binomial in $P(\mathcal{S})$ this monomial is the trailing one.

Therefore the binomial $T_{i} T^{\alpha}-T_{j} T^{\beta}$ reduces to 0 by a sequence of binomials of degree 2. A binomial $T_{i} T_{k}-T_{l} T_{m}$ with $T_{i} T_{k}$ as initial term is not allowed since in that case either $l$ or $m$ should be equal to $j$ and this contradict the hypothesis.

Therefore the only binomials that reduce $T_{i} T^{\alpha}$ are $T_{\alpha(1)} T_{\alpha(2)}-T_{\gamma(1)} T_{\gamma(2)}$, with $T_{\alpha(1)} T_{\alpha(2)}$ that divides $T^{\alpha}$. We have

$$
T_{i} T^{\alpha}-T_{j} T^{\beta} \longrightarrow T_{i} \frac{T^{\alpha}}{T_{\alpha(1)} T_{\alpha(2)}} T_{\gamma(1)} T_{\gamma(2)}-T_{j} T^{\beta}
$$

We observe that $T_{i} \frac{T^{\alpha}}{T_{\alpha(1)} T_{\alpha(2)}} T_{\gamma(1)} T_{\gamma(2)}$ is the initial term of

$$
T_{i} \frac{T^{\alpha}}{T_{\alpha(1)} T_{\alpha(2)}} T_{\gamma(1)} T_{\gamma(2)}-T_{j} T^{\beta},
$$

if neither $\gamma(1)$ nor $\gamma(2)$ is equal to $j$. Then we continue the reduction steps, we say $k$ time, when $\gamma(2 k)=j$. Therefore we have $u_{i} u^{\alpha} \cong u_{i} \frac{u^{\alpha}}{u_{\alpha(1)} u_{\alpha(2)}} u_{\gamma(1)} u_{\gamma(2)} \cong \cdots$

$$
\cong u_{i} \frac{u^{\alpha}}{u_{\alpha(1)} \cdots u_{\alpha(2 k)}} u_{\gamma(1)} \cdots u_{\gamma(2 k-1)} u_{j} \cong u_{j} u^{\beta}
$$

and this implies $u_{i} u_{j}$ divides $u_{i} u^{\alpha} \cong u_{j} u^{\beta}$.

Case 2) Suppose that there are primitive binomials of degree 2 such that $T_{i} T_{k}-T_{j} T_{l} \in P(\mathcal{S})$. We want to show that

$$
\left(u_{i}\right) \cap\left(u_{j}\right)=\left(u_{i} u_{j}, u_{i} u_{k(1)} \cong u_{j} u_{l(1)}, \ldots, u_{i} u_{k(s)} \cong u_{j} u_{l(s)}\right) .
$$

Let $u_{i} u^{\alpha} \cong u_{j} u^{\beta}$ of degree $>2$. As in case 1) it follows $T_{i} T^{\alpha}-T_{j} T^{\beta} \in P(\mathcal{S})$ and, if we fix a degree reverse lexicographic ordering such that $T_{j}<T_{i}<T_{k}$ $\forall k=1, \ldots, r, k \neq i, k \neq j$, then the Gröbner basis of $P(\mathcal{S})$ has degree 2 .

Therefore the binomial $T_{i} T^{\alpha}-T_{j} T^{\beta}$ reduces to 0 by a sequence of binomials of degree 2.

After a number of steps, we say $k$, we have either as in case 1) a sequence of binomials

$$
T_{\alpha(1)} T_{\alpha(2)}-T_{\gamma(1)} T_{\gamma(2)}, \ldots, T_{\alpha(2 k-1)} T_{\alpha(2 k)}-T_{\gamma(2 k-1)} T_{\gamma(2 k)}
$$

with $\gamma(2 k)=j$ then $u_{i} u_{j}$ divides $u_{i} u^{\alpha}$, or we'll have

$$
T_{\alpha(1)} T_{\alpha(2)}-T_{\gamma(1)} T_{\gamma(2)}, \ldots, T_{\alpha(2 k-1)} T_{\alpha(2 k)}-T_{\gamma(2 k-1)} T_{\gamma(2 k)}
$$


with $\alpha(2 k)=i$ and since $T_{\alpha(2 k-1)} T_{\alpha(2 k)}=T_{\alpha(2 k-1)} T_{i}$ is the initial term it implies, since $T_{i}<T_{k}$ with $k \neq j$, that $\gamma(2 k)=j$.

Therefore we have that $T_{\alpha(2 k-1)} T_{i}-T_{\gamma(2 k-1)} T_{j}$ are related with the minimal generators $u_{\alpha(2 k-1)} u_{i} \cong u_{\gamma(2 k-1)} u_{j}$.

So

$$
\begin{gathered}
u_{i} u^{\alpha} \cong u_{i} \frac{u^{\alpha}}{u_{\alpha(1)} u_{\alpha(2)}} u_{\gamma(1)} u_{\gamma(2)} \cong \cdots \cong u_{i} \frac{u^{\alpha}}{u_{\alpha(1)} \cdots u_{\alpha(2 k-2)}} u_{\gamma(1)} \cdots u_{\gamma(2 k-2)} \cong \\
u_{j} \frac{u^{\alpha}}{u_{\alpha(1)} \cdots u_{\alpha(2 k-1)}} \frac{u_{i}}{u_{\alpha(2 k)}} u_{\gamma(1)} \cdots u_{\gamma(2 k-1)}
\end{gathered}
$$

and this implies $u_{\alpha(2 k-1)} u_{i} \cong u_{\gamma(2 k-1)} u_{j}$ divides $u_{i} u^{\alpha} \cong u_{j} u^{\beta}$.

\section{Veronese rings and Segre product of poly- nomials rings}

In this section we prove that the following classes of homogeneous semigroup rings:

1. The 2th Veronese algebras;

2. The Segre products of two polynomial rings;

are strongly Koszul and their toric ideals admit a universal reverse lexicographic Gröbner basis of degree 2.

(1) Let $R$ the second Veronese subring of $K\left[x_{1}, \ldots, x_{n}\right]$. We write $R=$ $K\left[x_{i} x_{j}: 1 \leq i \leq j \leq n\right]$ and consider its presentation

$$
0 \longrightarrow P(\mathcal{S}) \longrightarrow S=K\left[T_{i j}: 1 \leq i \leq j \leq n\right] \longrightarrow R
$$

We say $N=n(n+1) / 2$ the number of variables of $S$.

If we fix the degree reverse lexicographic ordering on $S$, with respect to one of the $N$ ! degree reverse lexicographic ordering induced by all permutations of the variables $T_{i j}, 1 \leq i \leq j \leq n$, then the ideal $P(\mathcal{S})$ is generated by the following set of binomials, $\forall i_{1}, i_{2}, i_{3}, i_{4} \in\{1, \ldots, n\}, G=G_{1} \cup G_{2} \cup G_{3}$

$$
\begin{gathered}
G_{1}=\left\{T_{i_{1} i_{3}} T_{i_{2} i_{4}}-T_{i_{1} i_{2}} T_{i_{3} i_{4}}, T_{i_{1} i_{4}} T_{i_{2} i_{3}}-T_{i_{1} i_{2}} T_{i_{3} i_{4}}, T_{i_{1} i_{3}} T_{i_{2} i_{4}}-T_{i_{1} i_{4}} T_{i_{2} i_{3}} \mid\right. \\
\left.\#\left\{i_{1}, i_{2}, i_{3}, i_{4}\right\}=4\right\}
\end{gathered}
$$




$$
\begin{aligned}
& G_{2}=\left\{T_{i_{1} i_{4}} T_{i_{2} i_{3}}-T_{i_{1} i_{2}} T_{i_{3} i_{4}} \mid \text { where either } i_{1}=i_{2} \text { or } i_{2}=i_{3} \text { or } i_{3}=i_{4}\right. \text { and } \\
& \left.\quad \#\left\{i_{1}, i_{2}, i_{3}, i_{4}\right\}=3\right\} ; \\
& G_{3}=\left\{T_{i_{1} i_{3}}^{2}-T_{i_{1} i_{1}} T_{i_{3} i_{3}}-\#\left\{i_{1}, i_{2}, i_{3}, i_{4}\right\}=2 \text { and } i_{1}=i_{2}, i_{3}=i_{4}\right\} \text { (in the } \\
& \quad \text { other cases the binomial is a product of } 2 \text { linear forms). }
\end{aligned}
$$

Definition 3.1. A monomial $u=T_{i_{1} i_{2}} T_{i_{3} i_{4}} \cdots T_{i_{r-1} i_{r}} \in S$ is ordered if

$$
T_{i_{k} i_{k+1}} \leq T_{i_{l} i_{l+1}} \quad \forall k=1, \ldots, r-1, \quad \forall\left\{i_{l}, i_{l+1}\right\} \subset\left\{i_{1}, \ldots, i_{k+1}\right\}
$$

Remark 3.2. It is easy to observe that if we fix the degree reverse lexicographic order induced by

$$
T_{11}>T_{12}>\cdots>T_{1 n}>T_{22}>\cdots>T_{2 n}>\cdots>T_{n n}
$$

then a monomial $u=T_{i_{1} i_{2}} T_{i_{3} i_{4}} \cdots T_{i_{r-1} i_{r}} \in S$ is ordered if

$$
i_{1} \leq i_{2} \leq i_{3} \leq i_{4} \leq \cdots \leq i_{r-1} \leq i_{r}
$$

Definition 3.3. Let $u=T_{i_{1} i_{2}} T_{i_{3} i_{4}} \cdots T_{i_{r-1} i_{r}}$ a monomial in $S$. We call the set

$$
\left\{i_{1}, i_{2}, \ldots, i_{r}\right\}
$$

the index support of $u$.

Remark 3.4. If $u-v$ is a binomial in $P(\mathcal{S})$, it is easy to show that $u$ and $v$ have the same index support.

Proposition 3.5. The following facts are true:

1. The ordered monomial is the minimum monomial with a given index support;

2. In every case the trailing term of a binomial in $G$ is ordered. The only exception is one of the 3 binomial of the set $G_{1}$. In this case this binomial is reducible by the division algorithm to 0 , by the other two binomials of $G_{1}$

3. A monomial $T_{i_{1} i_{2}} T_{i_{3} i_{4}}$ that is not ordered, is reducible by one of the binomials in $G$ such that the reduced monomial is an ordered one. 
Proof. (1) Let

$$
\left\{i_{1}, i_{2}, \ldots, i_{r}\right\}
$$

be the given index support and suppose that

$$
T_{i_{r-1} i_{r}} \leq T_{i_{l} i_{l+1}}, \quad \forall\left\{i_{l}, i_{l+1}\right\} \subset\left\{i_{1}, \ldots, i_{r}\right\}
$$

All the monomials with a given index support that are divisible by $T_{i_{r-1} i_{r}}$ are less than the other monomial with the same index support. We call this set of monomials $H$. Now suppose that

$$
T_{i_{r-3} i_{r-2}} \leq T_{i_{l} i_{l+1}} \quad \forall\left\{i_{l}, i_{l+1}\right\} \subset\left\{i_{1}, \ldots, i_{r-2}\right\}
$$

All the monomials in $H$ that are divisible by $T_{i_{r-3} i_{r-2}}$ are minimal in this set, and so on. At the end is easy to observe that the ordered monomial is the minimum with a given index support. (2) and (3) are easy.

Now is possible to prove the following

Theorem 3.6. The set $G$ is a universal degree reverse lexicographic Gröbner basis.

Proof. Let $f_{1}, f_{2}$ be two binomials of $G$, with initial terms not coprime.

The s-pair $S\left(f_{1}, f_{2}\right)=u-v$ is a binomial of degree 3 . The index-support of $u$ and $v$ is $\left\{i_{1}, i_{2}, i_{3}, i_{4}, i_{5}, i_{6}\right\}$.

Let $u$ the initial term of $u-v$. This implies that $u$ is not ordered. Therefore there exists a binomial in $G$ whose initial term is not ordered that divides $u$ whose reduction have 4 of the 6 indexes ordered. We reduce $u-v$ by this binomial and obtain a new binomial $u^{\prime}-v$. Now, either $u^{\prime}$ or $v$ is the initial term, and this implies that this term is not ordered. After a finite number of reduction of this kind, we obtain that $u^{\prime \prime}$ and $v^{\prime \prime}$ are ordered and since they have the same index-support they are equal.

(2) Segre product of two polynomial rings.

Let $S_{1}=K\left[x_{1}, \ldots, x_{n}\right]$ and $S_{2}=K\left[y_{1}, \ldots, y_{m}\right]$. The Segre product $S_{1} * S_{2}$ is $R=K\left[x_{i} y_{j}: i=1, \ldots, n ; j=1, \ldots, m\right]$ and its presentation is given by

$$
0 \longrightarrow P(\mathcal{S}) \longrightarrow S=K\left[T_{i j}: i=1, \ldots, n, j=1, \ldots, m\right] \longrightarrow R \longrightarrow 0
$$


The ideal $P(\mathcal{S})$ is generated by the following binomials:

$$
G=\left\{T_{i_{1} j_{2}} T_{i_{2} j_{1}}-T_{i_{1} j_{1}} T_{i_{2} j_{2}}: i_{1} \neq i_{2} \in\{1, \ldots, n\}, j_{1} \neq j_{2} \in\{1, \ldots, m\}\right\}
$$

We fix the degree reverse lexicographic ordering on $S$ with respect to one of the $(\mathrm{nm})$ ! degree reverse lexicographic order induced by all permutations of the variables $T_{i j}, 1 \leq i \leq n, 1 \leq j \leq m$.

Definition 3.7. A monomial $u=T_{i_{1} j_{1}} T_{i_{2} j_{2}} \cdots T_{i_{r} j_{r}} \in S$ is ordered if

$$
T_{i_{k} j_{k}} \leq T_{i_{l} j_{l}} \quad \forall k=1, \ldots, r, \forall i_{l} \in\left\{i_{1}, \ldots, i_{k}\right\}, \forall j_{l} \in\left\{j_{1}, \ldots, j_{k}\right\}
$$

Remark 3.8. Even in this case happens that:

1. The ordered monomial is the minimum monomial with a given index support.

2. A monomial $T_{i_{1} j_{1}} T_{i_{2} j_{2}}$ that is not ordered, is reducible by one of the binomials in $G$ such that the reduced monomial is an ordered one.

Now is possible to prove the following

Theorem 3.9. The set $G$ is a universal degree reverse lexicographic Gröbner basis.

Proof. Let $f_{1}, f_{2}$ be two of the binomials in $G$, with initial terms that are not coprime.

The s-pair $S\left(f_{1}, f_{2}\right)=u-v$ is a binomial of degree 3 . The index-support of $u$ and $v$ is $\left\{i_{1}, i_{2}, i_{3}, i_{4}, i_{5}, i_{6}\right\}$.

Let $u$ the initial term of $u-v$. This implies that $u$ is not ordered. Therefore there exists a binomial in $G$ whose initial term is not ordered that divides $u$ whose reduction have 4 of the 6 indexes ordered. We reduce $u-v$ by this binomial and obtain a new binomial $u^{\prime}-v$. Now, either $u^{\prime}$ or $v$ is the initial term, and this implies that this term is not ordered. After a finite number of reduction of this kind, we obtain that $u^{\prime \prime}$ and $v^{\prime \prime}$ are ordered and since they have the same index-support they are equal.

Remark 3.10. The set $G$ in Theorem 3.9 is a universal degree reverse lexicographic, but not a universal Gröbner basis. 
In fact, if we consider the Segre product

$$
\begin{aligned}
& K\left[x_{1}, x_{3}, x_{5}\right] * K\left[x_{2}, x_{4}, x_{6}\right] \cong \\
& K\left[x_{1} x_{2}, x_{1} x_{4}, x_{1} x_{6}, x_{2} x_{3}, x_{2} x_{5}, x_{3} x_{4}, x_{5} x_{6}, x_{4} x_{5}, x_{3} x_{6}\right]
\end{aligned}
$$

and its presentation

$0 \longrightarrow P(\mathcal{S}) \longrightarrow S=K\left[y_{1}, \ldots, y_{9}\right] \longrightarrow$

$$
K\left[x_{1} x_{2}, x_{1} x_{4}, x_{1} x_{6}, x_{2} x_{3}, x_{2} x_{5}, x_{3} x_{4}, x_{5} x_{6}, x_{4} x_{5}, x_{3} x_{6}\right] \longrightarrow 0
$$

we obtain that $P(\mathcal{S})$ is generated by the forms

$y_{1} y_{6}-y_{2} y_{4}, y_{1} y_{8}-y_{2} y_{5}, y_{4} y_{8}-y_{5} y_{6}, y_{1} y_{9}-y_{3} y_{4}, y_{2} y_{9}-y_{3} y_{6}$,

$$
y_{1} y_{7}-y_{3} y_{5}, y_{5} y_{9}-y_{4} y_{7}, y_{3} y_{8}-y_{2} y_{7}, y_{8} y_{9}-y_{6} y_{7} \text {. }
$$

Fixing a degree Lexicographic ordering induced by

$$
y_{9}>y_{8}>\cdots>y_{1}
$$

we obtain the S-polynomial

$$
S\left(y_{1} y_{6}-y_{2} y_{4}, y_{1} y_{7}-y_{3} y_{5}\right)=y_{2} y_{4} y_{7}-y_{3} y_{5} y_{6}
$$

that cannot be reduced by forms of degree two.

As a consequence of the above results, we formulate the following

Conjecture 3.11. Let $K[\mathcal{S}]$ a homogeneous semigroup ring. The following facts are equivalent:

1. $K[\mathcal{S}]$ is a strongly Koszul algebra;

2. $P(\mathcal{S})$ has a universal degree reverse lexicographic Gröbner basis of degree 2.

\section{References}

[1] A. Aramova - J. Herzog - T. Hibi, Finite lattices and lexicographic Gröbner bases European J. combin. 21 (2000), 431-439.

[2] W. Bruns and J. Herzog, Cohen-Macaulay Rings, Cambridge University Press, Cambridge, Revised Edition, 1997.

[3] W. Bruns, J. Herzog, Udo Vetter Syzygies and Walks, Commutative Algebra (Aron Simis, Ngo Viet Trung, and Giuseppe Valla, eds.), World Scientific, 1994, 14-25 September 1992, ICTP, Trieste, Italy, pp. 36-57. 
[4] A. Capani, G. Niesi and L. Robbiano, CoCoA: A system for doing computations in commutative algebra. 1998. Available via anonymous ftp from lancelot.dima.unige.it.

[5] R. Fröberg An introduction to Gröbner bases Pure and applied mathematics, J. Wiley Sons, 1998.

[6] J.Herzog - T. Hibi - G. Restuccia, Strongly Koszul algebras Math. Scand. $86,161-178,(2000)$

intersections

[7] B. Sturmfels, Gröbner Bases and Convex Polytopes, University Lecture Series 8, American Mathematical Society, Rhode Island, 1996.

[8] R.H. Villarreal. Monomial Algebras. Pure and applied mathematics. Marcel Dekker, 2001.

Received: July 10, 2006 